\title{
Nova Acta Leopoldina: lebende Dokumente als neues Publikationsmodell
}

DIETHARD TAUTZ

MAX-PLANCK-INSTITUT FÜR EVOLUTIONSBIOLOGIE, PLÖN
DOI: 10.1007/s12268-019-1014-1

(C) Springer-Verlag 2019

\begin{abstract}
Alles Wissen und alle Vermehrung unseres Wissens endet nicht mit einem Schlusspunkt, sondern mit einem Fragezeichen
\end{abstract}

(Hermann Hesse)

Zweifellos würde jeder Wissenschaftler dieser Feststellung zustimmen. Aber wenn wir Wissenschaft publizieren, dann halten wir uns bisher nicht daran. Denn jedes wissenschaftliche Dokument soll möglichst mit „einem Schlusspunkt“ enden, das heißt, es soll eine Version erstellt werden, die nicht mehr verändert wird. Das erleichtert die Archivierung und erlaubt Verlagsrechte $\mathrm{zu}$ sichern. Aber es ist nicht das Wesen der Wissenschaft, die sich ja kontinuierlich weiterentwickelt. Warum sollten sich nicht auch Dokumente weiterentwickeln?

Die Nationale Akademie der Wissenschaften Leopoldina hat jetzt beschlossen, ihre Publikationen in ein neues Zeitalter zu führen. Mit der seit 1670 existierenden Zeitschrift Nova Acta Leopoldina (NAL; früher unter anderen Titeln) gibt sie die älteste wissenschaftliche Zeitschrift der Welt heraus. Diese soll jetzt nicht nur dem Open-AccessZeitalter angepasst werden, sondern es wird auch ein neues

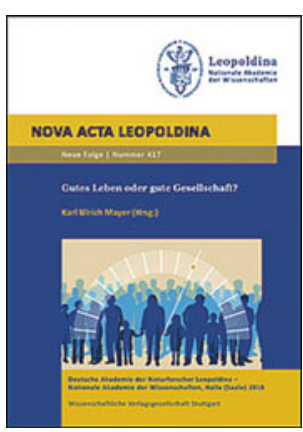

te man das also nicht auch generell für

Insbesondere bei Reviews ist doch klar, dass es keinen so großen Sinn macht, ein völlig neues Dokument zu schreiben, wenn ein bestehendes einfach fortgeschrieben werden könnte. Tatsächlich gibt es ein solches System bereits in der Physik (z. B. die Living Reviews in Relativity), hier sind es aber vor allem die ursprünglichen Autoren selbst, die es weiterschreiben. Es könnte jedoch - ähnlich wie bei Wikipedia - auch zu einer Gemeinschaftsaufgabe von Wissenschaftlern werden. Im Gegensatz zu Wikipedia muss dies allerdings einer Kontrolle unterliegen, in der Editoren und Peer-Reviewer die Qualität sicherstellen.

Das „Leben“ eines solchen Manuskripts beginnt wie üblich: Ein ausgewiesener Wissenschaftler oder eine Wissenschaftlerin erstellt ein klassisches Manuskript. Dieses wird einem Peer-Review unterworfen und dann als Version 1.0 ins Netz gestellt, die dann kommentiert werden kann. Änderungsvorschläge oder neue Entwicklungen können in einem aktiven Prozess eingebracht werden. Editoren überwachen den Prozess und schlagen ab einem sion $2.0 \mathrm{zu}$ generieren. Diese kann vom ursprünglichen Autor bzw. der Autorin erstellt werden oder auch von einem neuen und dieses soll dann unter dem Titel NAL-live erscheinen.

Das Modell sieht „lebende Dokumente“ vor, die kontinuierlich weitergeschrieben werden können. Die Idee ist eigentlich nicht neu. Bücher wurden und werden oft bei Neuauflagen korrigiert und/oder erweitert, lexikalische Werke werden weitergeschrieben, und Wikipedia hat die internetbasierte Erstellung und Fortschreibung von Texten zu einer Gemeinschaftsaufgabe gemacht. Warum soll-

Autor oder einer neuen Autorin. Da alle sionen einer freien Lizensierung nach dem CC-BY-4.0-Modell (Wiederverwendung und Veränderung frei, unter Angabe der Quelle) unterliegen, können neue Autoren problemlos Textteile und Bilder früherer Versionen weiterhin verwenden. Ein solches Dokument kann damit potenziell über Jahrzehnte „weiterleben“.

Damit werden solche Texte zu einer GenePublikationsmodell entwickelt, wissenschaftliche Texte einführen? bestimmten Punkt vor, eine Ver-

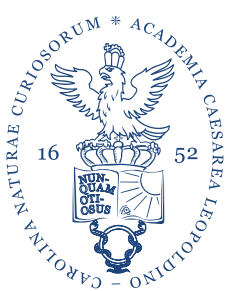

Leopoldina

Nationale Akademie der Wissenschaften letztlich nur von langlebigen wissenschaftlichen Institutionen gesichert werden, z. B. einer wissenschaftlichen Akademie wie der Leopoldina. Gleichzeitig ändert sich die Rolle von Autoren von einer Verbindung zu einzelnen Werken unter ihrem Namen zu einer Gemeinschaftsaufgabe. Ein wichtiger Bestandteil des Systems wird es daher sein, dass die Beiträge aller Autoren und Kommentatoren festgehalten werden, sodass diese sie auch als Teil ihrer wissenschaftlichen Leistungen nachweisen können.

NAL-live ist international angelegt und wird in Englisch im Gold-Open-Access-Modell erscheinen. Zu den Gründungseditoren gehören Diethard Tautz (Leitung) sowie jeweils ein Vertreter der vier Klassen der Leopoldina (Gerd Leuchs für die Mathematik, Natur- und Technikwissenschaften, Alfred Wittinghofer für die Lebenswissenschaften, Ulf Eysel für die Medizin und Christine Windbichler für die Geistes-, Sozial- und Verhaltenswissenschaften). Diese stellen derzeit das editorial board zusammen, das auch internationale Mitglieder der Leopoldina umfassen wird. Die ersten Dokumente sollen sich vor allem mit Themen von hohem öffentlichen Interesse beschäftigen. Sie sollen möglichst so geschrieben werden, dass sie auch einer interessierten Öffentlichkeit zugänglich sind. Damit sollen die Dokumente auch Grundlagen für politische Entscheidungsprozesse bilden. Potenzielle Autoren können sich gerne bei den Editoren melden.



sse:

Prof. Dr. Diethard Tautz Max-Planck-Institut für Evolutionsbiologie Abt. Evolutionsgenetik August-Thienemann-Straße 2 D-24306 Plön tautz@evolbio.mpg.de www.mpil-ploen.mpg.de 